\title{
Clinical Medicine Insights: Pathology
}

\section{Molecular Insights into Systemic Lupus Erythematosus Pathogenesis}

\section{Dama Laxminarayana}

Editor in Chief, Clinical Medicine Insights: Pathology, Formerly with Section on Rheumatology and Immunology, Department of Internal Medicine, Wake Forest University School of Medicine, Winston-Salem, NC, USA.

KEYWORDS: systemic lupus erythematosus, RNA editing, transcriptome, gene mutations, deamination, auto-antigens, auto-antibodies, autoimmunity

CITATION: Laxminarayana. Molecular Insights into Systemic Lupus Erythematosus Pathogenesis. Clinical Medicine Insights: Pathology 2014:7 7-9 doi: $10.4137 /$ CPath.S14814

TYPE: Editorial

FUNDING: Author discloses no funding sources.

COMPETING INTERESTS: Author discloses no potential conflicts of interest.

COPYRIGHT: $\odot$ the authors, publisher and licensee Libertas Academica Limited. This is an open-access article distributed under the terms of the Creative Commons CCBY-NC 3.0 License.

CORRESPONDENCE: laxmina@triad.rr.com

Systemic lupus erythematosus (SLE) is a complex, heterogeneous, and chronic autoimmune disorder of an unknown origin. Its clinical symptoms range from a benign skin disorder to severe, life-threatening conditions. ${ }^{1}$ Immune effector dysfunctions are hall marks of SLE disease. ${ }^{2}$ The etiopathogenesis of the altered immune response in SLE remains unknown. SLE is characterized by the presence of auto-antibodies (AutoAbs) for a wide variety of self antigens and circulating immune complexes. ${ }^{1,2}$ The onset of lupus is variable and may affect all stages of life. The disease predominantly afflicts females in the child-bearing years about 6- to 10-fold more frequently than males. We have not had a new drug in 50 years, because of the unknown etiology of the abnormal immune response. The current lupus therapies are non-specific, symptomatic, and cause significant side effects. In this editorial, I have made an attempt to describe multistep immune alterations that pave the way for the inception and sustaining of SLE pathogenesis, and postulated molecular mechanisms involved in SLE disease onset. Such information will help in better understanding SLE etiopathogenesis and in developing effective and safer strategies to combat SLE as well as other autoimmune diseases.

A strong association has been found between elevated levels of circulating type I interferons (IFNs) and autoimmune diseases like type I diabetes and SLE. ${ }^{3-6}$ Remarkably, the therapeutic administration of type I IFNs has provoked type I diabetes, SLE, and primary Sjögren's syndrome $(\mathrm{pSS})$ in some individuals. ${ }^{7-9}$ Constitutive expression of type I IFNs was observed in SLE patients. ${ }^{5}$ The expression and up-regulation of several type I IFN-regulated genes is associated with SLE pathogenesis and disease severity. ${ }^{10-12}$ Natural IFN- $\alpha$ producing cells (NIPCs)/plasmacytoid dendritic cells ( $\mathrm{pDCs}$ ) play a major role in endogenous type I IFN production and NIPCs/pDCs are increased in SLE. ${ }^{13}$ Deficient expression of the type I IFN receptor reduced lupus-like disease in NZB mice. ${ }^{14}$ These studies demonstrate strong correlation between type I IFNs and SLE pathogenesis. In seeking the molecular mechanism(s) for lupus pathogenesis, I discovered editing in SLE T cell transcriptome, ${ }^{15}$ because of the up-regulation of the transcript editing gene, $150 \mathrm{kDa}$ adenosine deaminase that act on RNA 1 (ADAR1). ${ }^{16}$ Studies by other investigators confirmed these findings. ${ }^{10,17,18}$ The ADARs belong to a family of mammalian RNA editing enzymes, which play an important role in several physiological and pathological processes by catalyzing hydrolytic deamination at C-6 of the adenosine (A) base in certain mRNAs, which leads to inosine (I) formation. ${ }^{15,16,19}$ Inosines are subsequently recognized as guanosine $(G)$ by the translation machinery. Such editing will result in A to $I(G)$ transcript mutation. The $150 \mathrm{kDa}$ ADAR 1 expression is regulated by type I IFNs while 110-kDA ADAR1 and ADAR2 are constitutively expressed in $T$ cells and other cell types.

The occurrence of conserved RNA secondary structures in the human transcriptome is extensive, ${ }^{20}$ which indicates enormous amounts of potential ADAR substrates in the human transcriptome. Widespread ADARs mediated editing of exonic and intronic elements in human RNAs has been identified. ${ }^{21-28}$ Most of the editing of exonic elements is random and 
heterogeneous. ${ }^{15,16}$ The up-regulated $150 \mathrm{kDa}$ ADAR1 randomly edit adenosines located in double stranded base-paired coding and noncoding regions and cause novel mutations in gene transcripts. ${ }^{16,24,25}$ Repeated occurrence of such exonic and intronic editing at certain base positions has been identified in SLE T cells and in normal T cells, which express up-regulated $150 \mathrm{kDa}$ ADAR1, at different time points. ${ }^{16,24-26}$ In addition to ADARs induced A to I $(\mathrm{G})$ editing, apolipoprotein B-editing enzyme, catalytic polypeptide-1 (APOBEC1) mediated cytidine (C) to uridine (U) editing has been well documented in human transcriptome. ${ }^{29} \mathrm{~A}$ low frequency of $\mathrm{G}$ to $\mathrm{A}$ and $\mathrm{U}$ to $\mathrm{C}$ changes were also observed only in SLE and other pathological conditions. ${ }^{24}$ The enzymatic machinery responsible for such editing and the molecular mechanism underlying such changes are unknown. Protein molecules translated from edited mRNAs have been identified in normal human B-lymphocytes. ${ }^{30}$ Recently, about hundred million A to I editing sites were identified in human transcriptome, ${ }^{28}$ which can make possible a generation of extremely diverse transcriptome. Extensive editing of human genome by activation-induced cytidine deaminase (AID) enzyme has been well documented. ${ }^{31,32}$ The AID mediated DNA editing may result in the formation of anti-DNA antibodies in addition to its role in the induction of somatic hyper mutations and class switch recombinations in immunoglobulin genes of B-lymphocytes. The occurrence of AutoAbs for mutant DNA molecules in scleroderma has been described recently. ${ }^{33}$ Peptidylarginine deiminases (PADs) edit protein molecules by deiminating arginine into citrulline and play a critical role in generating anti-citrulline antibodies in rheumatoid arthritis (RA). The association of anti-citrulline antibodies with RA pathogenesis is well established. ${ }^{34}$ The autoAbs to several proteins, RNA molecules were identified in addition to anti-DNA antibodies in SLE. Occurrence of such plethora of autoAbs in SLE by molecular mimicking (self antigens mimicking as viral and/or bacterial products) without alterations, such as editing and/or mutations in DNA, RNA, and protein molecules is impossible. Therefore, it is hypothesized that, altered and/or enhanced DNA, RNA, and protein editing will not only induce altered gene regulations and immune functions but also set the stage for production of novel auto-antigens (autoAgs). The occurrence of such process repeatedly at different time points will result in the generation of autoAbs followed by auto-immunogenicity and the onset of autoimmunity.

The induction of autoimmunity involves two distinct phases. In the first phase autoAgs are formed by the following molecular mechanisms: (a) modulation of DNA, RNA, and proteins by editing and/or by induction of somatic mutations; (b) occurrence of same editing and/or mutation(s) at specific site(s) at different time points; (c) apoptosis of cells carrying such editing and/or mutation(s) and impaired clearance of apoptotic material by nucleases and proteases; and (d) presentation of such altered DNA, RNA, and protein molecules as non self by antigen presenting cells to $T$ cells. Type I IFNs and/or IFN-inducible genes in the presence of autoAgs, will promote the activation and survival of naive $\mathrm{T}$ cells by dendritic cells (DCs), which is independent of $\mathrm{BCL}$ and $\mathrm{BCL}_{\mathrm{XL}}$ gene function. ${ }^{35}$ Activated $\mathrm{T}$ cells will induce $\mathrm{B}$ cell stimulation and production of autoAbs. ${ }^{36}$ This process needs constitutive and repeated occurrence of specific editing and/or mutations at the same site(s) in DNA, RNA, and/or proteins followed by impaired cellular functions and auto-immunogenicity as described earlier. Such initiated autoimmunity will be sustained by the following events, which occur as second phase; (a) the autoimmune complexes formed in the first phase act as endogenous inducers of type I IFNs, replacing exogenous type I IFNs and continuously inducing the production of type I IFNs by NIPCs; (b) continuous generation of autoAbs and autoimmune complexes is maintained this process; (c) a vicious cycle becomes established ${ }^{5}$; (d) in addition, superantigens (SAgs), products of type I IFN-regulated HERVs, target the immune system causing massive polyclonal $T$ cell activation, cytokine release, $\mathrm{T}$ cell apoptosis, and/or anergy, which aid in enhancing autoimmunity. ${ }^{37}$ Therefore, such information indicates why lupus pathogenesis is so complex, variable, and hard to predict definite cause(s) for and raise the following questions. During their life time, all individuals will sustain viral infections, which are combated by endogenous and exogenous IFNs and IFN-regulated genes. However, why do only relatively few people develop autoimmunity, especially certain women during the childbearing years? Why do only a small percentage of cancer patients (20\%), who are treated with IFN, express transient autoimmunity and only a fraction of them (1\%) acquire SLE? ${ }^{7}$ Why do about $20 \%$ of normal subjects demonstrate the presence of antinuclear antibodies (ANAs) but fail to develop the onset of autoimmunity and why do age related increase occur in the prevalence of AutoAbs in healthy elderly subjects? ${ }^{38}$ These questions will help in hypothesizing that only some editing events and/or mutations in DNA, RNA, and protein will result in the formation of autoAgs, like how only extremely rare somatic mutations initiate cancer induction. These autoAgs will be able to produce autoAbs and induce autoimmunity only when cells containing such autoAgs undergo apoptosis followed by non clearance of apoptotic material. In addition, the induction of autoimmunity mimics the process of immunization, which needs vaccination with pathogenic material followed by repeated booster dose administration to attain good immune response for such pathogens. This may also be true in the process of attaining autoimmunity, in which repeated production of autoAbs to specific edited and/or mutated DNA, RNA, and protein molecules and their availability for developing auto-immunogenicity are important and necessary.

Based on this information, I postulate that, no present and/or future drug(s) will help in curing and/or preventing autoimmunity, specifically SLE after its onset, except for symptomatic treatment and temporary relief. Drug therapy(s) cannot modulate and/or suppress such a multistep and complex autoimmune response generated by altered plethora of self DNA, RNA, and protein molecules, before and after 
the onset of SLE pathogenesis. Moreover, it will be impossible to delineate the autoimmune response from normal immune response to selectively suppress it, without impairing normal immune response. Therefore, the best strategy to combat this anomaly is the multipronged approach of monitoring and regulating (a) frequent and prolonged expression of type I IFNs; (b) DNA, RNA, and protein editing; (c) apoptosis; (d) clearance of apoptotic material by nucleases and proteases during autoimmunity onset susceptible circumstances such as repeated viral and bacterial infections, radiation exposure, cancer treatment, and in women during child bearing years. Such timely and focused regimen and/or approaches could pave the way for effective and safer ways to prevent and/or control SLE as well as other autoimmune diseases.

\section{Author Contributions}

Conceived the concept: DL. Analyzed the data: DL. Wrote the first draft of the manuscript: DL. Made critical revisions: DL. Author reviewed and approved of the final manuscript.

\section{DISCLOSURES AND ETHICS}

As a requirement of publication the author has provided signed confirmation of compliance with ethical and legal obligations including but not limited to compliance with ICMJE authorship and competing interests guidelines, that the article is neither under consideration for publication nor published elsewhere, of their compliance with legal and ethical guidelines concerning human and animal research participants (if applicable), and that permission has been obtained for reproduction of any copyrighted material.

\section{REFERENCES}

1. Kotzin BL. Systemic lupus erythematosus. Cell. 1996;85:303-6.

2. Tsokos GC. Overview of cellular immune function in systemic lupus erythematosus. In: Lahita RG, ed. Systemic Lupus Erythematosus. New York: Churchill Livingstone; 1992:15-50.

3. Hooks JJ, Moutsopoulos HM, Geis SA, Stahl NI, Decker JL, Notkins AL. Immune interferon in the circulation of patients with autoimmune disease. NEnglJMed. 1979;301:5-8.

4. Foulis AK, Farquharson MA, Meager A. Immunoreactive alpha-interferon in insulin secreting beta cells in type I diabetes mellitus. Lancet. 1987;2:1423-7.

5. Rönnblom L, Alm GV. An etiopathogenic role for the type I IFN system in SLE. Trends Immunol. 2001;22:427-31.

6. Theofilopoulos AN, Baccala R, Beutler B, Kono DH. Type I interferons (alpha/ beta) in immunity and autoimmunity. Annu Rev Immunol. 2005;23:307-36.

7. Ronnblom LE, Alm GV, Oberg KV. Possible induction of systemic lupus erythematosus by interferon-alpha treatment in a patient with malignant carcinoid tumour. J Intern Med. 1990;227:207-10.

8. Ronnblom LE, Alm GV, Oberg KV. Autoimmunity after alpha-interferon therapy for malignant carcinoid tumors. Ann Intern Med. 1991;115:178-83.

9. Unoki H, Moriyama A, Tabaru A, Masumoto A, Otsuki M. Development of Sjogren's syndrome during treatment with recombinant human interferon-alpha2b for chronic hepatitis C. J Gastroenterol. 1996;31:723-7.

10. Crow MK, Wohlgemuth J. Microarray analysis of gene expression in lupus. Arthritis Res Ther. 2003;5:279-87.

11. Baechler EC, Batliwalla FM, Karypis G, et al. Interferon inducible gene expression signature in peripheral blood cells of patients with severe lupus. Proc Natl Acad Sci USA. 2003;100:2610-5.
12. Bennett L, Palucka AK, Arce E, et al. Interferon and granulopoiesis signature in systemic lupus erythematosus blood. JExp Med. 2003;197:711-23.

13. Rönnblom L, Alm GV. A pivotal role for the natural interferon alpha-producing cells (plasmacytoid dendritic cells) in the pathogenesis of lupus. J Exp Med. 2001;294:F59-63.

14. Raber WRS, Baccala R, Haraldsson KM, et al. Type-I interferon receptor deficiency reduces lupus like disease in NZB mice. J Exp Med. 2003;197:777-88.

15. Laxminarayana D, Kammer GM. Messenger RNA mutations of type I protein kinase A regulatory subunit alpha in T lymphocytes of a subject with systemic lupus erythematosus. Int Immunol. 2000;12:1521-9.

16. Laxminarayana D, Khan IU, Kammer GM. Transcript mutations of the alpha regulatory subunit of protein kinase A and up-regulation of the RNA editing gene transcripts in lupus T lymphocytes. Lancet. 2002;360:842-9.

17. Toyabe S, Kaneko U, Uchiyama M. Decreased DAP12 expression in natural killer lymphocytes from patients with systemic lupus erythematosus is associated with increased transcript mutations. JAutoimmun. 2004;23:371-8.

18. Kaneko U, Toyabe S, Hara M, Uchiyama M. Increased mutations of CD72 transcript in B-lymphocytes from adolescent patients with systemic lupus erythematosus. Pediatr Allergy Immunol. 2006;17:565-71.

19. Bass BL. RNA editing by adenosine deaminases that act on RNA. Annu Rev Biochem. 2002;71:817-46.

20. Pedersen JS, Bejerano G, Siepel A, et al. Identification and classification of conserved RNA secondary structures in the human genome. PLoS Comput Biol. 2006;2:e33.

21. Levanon EY, Eisenberg E, Yelin R, et al. Systematic identification of abundant A-to-I editing sites in the human transcriptome. Nat Biotechnol. 2004;22:1001-5.

22. Athanasiadis A, Rich A, Maas S. Widespread A-to-I RNA editing of Alu-containing mRNAs in the human transcriptome. PLoS Biol. 2004;2:1-15.

23. Kim DD, Kim TT, Walsh T, et al. Widespread RNA editing of embedded Alu elements in the human transcriptome. Genome Res. 2004;14:1719-25.

24. Laxminarayana D, O'Rourke KS, Maas S, Olorenshaw I. Altered and novel editing in adenosine deaminase that act on RNA (ADAR) 2 gene transcripts of systemic lupus erythematosus (SLE) T lymphocytes. Immunology. 2007;121:359-69.

25. Laxminarayana D, Khan IU, O'Rourke KS, Giri B. Induction of $150 \mathrm{kDa}$ Adenosine Deaminase that acts on RNA (ADAR1) gene expression in normal T lymphocytes by antiCD3+ antiCD28. Immunology. 2007;122:623-33.

26. OrlowskiRJ,O'RourkeKS,OlorenshawI,HawkinsGA,MaasS,LaxminarayanaD. Altered editing in cyclic nucleotide phosphodiesterase 8 A1 (PDE8 A1) gene transcripts of systemic lupus erythematosus (SLE) T lymphocytes. Immunology. 2008;125:408-19.

27. Li JB, Levanon EY, Yoon JK, et al. Genome-wide identification of human RNA editing sites by parallel DNA capturing and sequencing. Science. 2009;324:1210-3.

28. Bazak L, Haviv A, Barak M, et al. A-to-I RNA editing occurs at over a hundred million genomic sites, located in a majority of human genes. Genome Res.2014; 24:365-76.

29. Rosenberg BR, Hamilton CE, Mwangi MM, Dewell S, Papavasiliou FN. Transcriptome-wide sequencing reveals numerous APOBEC1 mRNA-editing targets in transcript 3' UTRs. Nat Struct Mol Biol. 2011;18:230-6.

30. Li M, Wang IX, Li Y, et al. Widespread RNA and DNA sequence differences in the human transcriptome. Science. 2011;333(6038):53-8.

31. Muramatsu M, Nagaoka H, Shinkura R, Begum NA, Honjo T. Discovery of activation-induced cytidine deaminase, the engraver of antibody memory. Adv Immunol. 2007;94:1-36.

32. Carmi S, Church GM, Levanon EY. Large-scale DNA editing of retrotransposons accelerates mammalian genome evolution. Nat Commun. 2011;2:519.

33. Joseph CG, Darrah E, Shah AA, et al. Association of the autoimmune disease scleroderma with an immunologic response to cancer. Science. 2014;343:152-7.

34. Darrah E, Giles JT, Ols ML, Bull HG, Andrade F, Rosen A. Erosive rheumatoid arthritis is associated with antibodies that activate PAD4 by increasing calcium sensitivity. Sci Transl Med. 2013;5:186ra65.

35. Marrack PJ, Kappler J, Mitchell T. Type I interferons keep activated T cells alive. JExp Med. 1999;189:521-9.

36. Crow MK. Mechanisms of T-helper activation and function in systemic lupus erythematosus. In: Kammer GM, Tsokos GC, eds. Lupus: Molecular and Cellular Pathogenesis. Totowa, NJ: Humana Press; 1999:231-56.

37. Sutkowski N, Conrad B, Thorley-Lawson DA, Huber BT. Epstein-Barr virus transactivates the human endogenous retrovirus HERV-K18 that encodes a super antigen. Immunity. 2001;15:579-89.

38. Rajczy K, Vargha P, Beregi E. Relationship between immunoglobulin levels and specific antibody titers in the elderly. Z Gerontol. 1986;19:158-61. 